\title{
Delineation of the cell-extrinsic apoptosis pathway in the zebrafish
}

\author{
PM Eimon ${ }^{1}$, E Kratz ${ }^{1}$, E Varfolomeev ${ }^{1}$, SG Hymowitz ${ }^{2}$, H Stern ${ }^{3}$, \\ $\mathrm{J} \mathrm{Zha}^{3}$ and A Ashkenazi ${ }^{\star, 1}$ \\ 1 Department of Molecular Oncology, Genentech Inc., 1 DNA Way, South San \\ Francisco, CA 94080, USA \\ 2 Department of Protein Engineering, Genentech Inc., 1 DNA Way, South San \\ Francisco, CA 94080, USA \\ ${ }^{3}$ Department of Pathology, Genentech, Inc., 1 DNA Way, South San Francisco, \\ CA 94080, USA \\ * Corresponding author: A Ashkenazi, Departments of Molecular Oncology, \\ Protein Engineering, and Pathology, Genentech Inc., 1 DNA Way, South San \\ Francisco, CA 94080, USA. Tel: + 650-225-1853; Fax: + 650-467-8195; \\ E-mail: aa@gene.com
}

Received 12.6.06; accepted 26.6.06; published online 04.8.06 Edited by G Melino

\begin{abstract}
The mammalian extrinsic apoptosis pathway is triggered by Fas ligand (FasL) and Apo2 ligand/tumor necrosis factor (TNF)related apoptosis-inducing ligand (Apo2L/TRAIL). Ligand binding to cognate receptors activates initiator caspases directly in a death-inducing signaling complex. In Drosophila, TNF ligand binding activates initiator caspases indirectly, through JNK. We characterized the extrinsic pathway in zebrafish to determine how it operates in a nonmammalian vertebrate. We identified homologs of FasL and Apo2LTRAIL, their receptors, and other components of the cell death machinery. Studies with three Apo2L/TRAIL homologs demonstrated that they bind the receptors zHDR (previously linked to hematopoiesis) and ovarian TNFR (ZOTR). Ectopic expression of these ligands during embryogenesis induced apoptosis in erythroblasts and notochord cells. Inhibition of ZHDR, ZOTR, the adaptor ZFADD, or caspase-8-like proteases blocked ligand-induced apoptosis, as did antiapoptotic Bcl-2 family members. Thus, the extrinsic apoptosis pathway in zebrafish closely resembles its mammalian counterpart and cooperates with the intrinsic pathway to trigger tissue-specific apoptosis during embryogenesis in response to ectopic Apo2L/TRAIL expression.

Cell Death and Differentiation (2006) 13, 1619-1630.

doi:10.1038/sj.cdd.4402015; published online 4 August 2006
\end{abstract}

Keywords: Apo2L/TRAIL; death receptor; caspase; TNF superfamily; hematopoiesis

Abbreviations: Apo2L/TRAIL, Apo2 ligand/TNF-related apoptosis-inducing ligand; c-FLIP, cellular FLICE inhibitory protein; CNS, central nervous system; CrmA, cytokine response modifier A; DcR, decoy receptor; DD, death domain; DED, death effector domain; DISC, death-inducing signaling complex; DL, death ligand; DN, dominant-negative; DR, death receptor; $E C D$, extracellular domain; EST, expressed sequence tags; FADD, Fas-associated DD; FasL, Fas ligand; EGFP, enhanced green fluorescent protein; HDR, hematopoietic death receptor; HEK, human embryonic kidney; h.p.f., hours postfertilization; HVEM, herpes virus entry mediator; ICM, intermediate cell mass; ISH, in situ hybridization; JNK, c-Jun N-terminal kinase; MO, morpholino oligonucleotide; NGFR, nerve growth factor receptor; ntl, no tail; OTR, ovarian TNFR; QPCR, quantitative PCR; RBI, rostral blood island; shh, sonic hedgehog; TNF, tumor necrosis factor; TNFR, TNF receptor; TRADD, TNFR-associated DD; TUNEL, terminal deoxynucleotidyl transferase-mediated dUTP nick end labeling

\section{Introduction}

Two distinct signaling mechanisms, known as the cell-intrinsic and cell-extrinsic pathways, control activation of the proapoptotic caspase family of cysteine proteases in mammals. ${ }^{1}$ The intrinsic pathway responds primarily to developmental cues or cellular damage and triggers apoptosis through the $\mathrm{Bcl}-2$ gene family and the initiator protease caspase- 9 . $^{2}$ The extrinsic pathway mediates apoptosis in response to Fas ligand (FasL) and Apo2L/TRAIL (Apo2 ligand or tumor necrosis factor (TNF)-related apoptosis-inducing ligand), which signal respectively through the death domain (DD)containing receptors Fas or death receptor (DR)4 and DR5. These receptors use the adaptor molecule Fas-associated DD (FADD) to recruit the initiator proteases caspase-8 and -10 into a death-inducing signaling complex (DISC), where they are activated. ${ }^{3,4}$ Initiator caspases in turn cleave and activate effector caspases such as caspase- $3,-6$ and -7 to carry out programmed cell death.

While components of the intrinsic pathway apparently exist in all metazoans, the extrinsic pathway is a more recent evolutionary development. No TNF or TNF receptor (TNFR) superfamily members have been found to date in Caenorhabditis elegans. In Drosophila, a single TNF ligand (Eiger) and its associated receptor (Wengen) induce apoptosis indirectly, by activating the caspase-9 homolog DRONC through the c-Jun N-terminal kinase (JNK) pathway; Drosophila homologs of caspase-8 (DREDD) and FADD do not appear to play a role in the extrinsic apoptosis pathway. ${ }^{5,6}$ DD-containing TNFRs have been reported exclusively in vertebrates, with examples in teleost (below), avian, ${ }^{7}$ and mammalian species. ${ }^{3}$ However, not all DD-containing TNFRs are dedicated activators of the extrinsic apoptosis pathway. For example, mammalian TNFR1 signals through the adaptor TNFR-associated DD (TRADD) and its principal role in vivo is NF- $\kappa$ B activation, which inhibits apoptosis. ${ }^{8}$ Only DR4, DR5, and Fas have DDs that directly bind FADD.

In mammals, the extrinsic pathway plays an important role in regulating the immune system. ${ }^{9}$ Studies with gene-knockout mice suggest that the extrinsic pathway also may be required during embryogenesis. Mice deficient in FADD, caspase-8, or the extrinsic pathway inhibitor c-FLIP (cellular FLICE inhibitory protein) all die in utero between embryonic days 10.5 and $12.5 .{ }^{10-13}$ However, other observations seem 
to indicate that the extrinsic pathway per se is not essential for embryonic development. Ex vivo whole-embryo culture rescues all embryonic defects in caspase-8 knockout mice. ${ }^{14}$ Moreover, mice deficient for FasL or Apo2L/TRAIL signaling complete embryogenesis without significant defects. ${ }^{15-18}$

To gain further insight into the evolution of the extrinsic apoptosis pathway and its importance during embryogenesis, we turned to the zebrafish (Danio rerio). Although known zebrafish expressed sequence tags (ESTs) appear to encode portions of apoptosis genes, ${ }^{19}$ the extrinsic pathway remains poorly understood in this model organism. Three putative TNF superfamily ligands have been reported, but their activities remain uncharacterized. ${ }^{20-22}$ Two DD-containing TNFRs also have been described: ovarian TNFR (zOTR), which is highly expressed in ovary, ${ }^{22}$ and hematopoietic DR (zHDR), which has been implicated in hematopoiesis. ${ }^{23,24}$ Neither zHDR nor zOTR have unambiguous sequence homology to specific mammalian TNFRs, and their cognate ligand(s) and signaling mechanism(s) remain unknown.

Here, we systematically define the extrinsic apoptosis pathway in zebrafish and show that it resembles its mammalian counterpart. Disruption of the extrinsic pathway does not impede zebrafish embryonic development. However, ectopic expression of Apo2L/TRAIL homologs triggers apoptosis in specific embryonic tissues through zHDR and zOTR. Ligand-induced apoptosis in these tissues requires both the extrinsic and intrinsic pathways.

\section{Results}

\section{Extrinsic apoptosis pathway homologs in zebrafish}

Zebrafish possess a single FasL homolog and four Apo2L/ TRAIL relatives (designated zDL1a, zDL1b, zDL2, and a

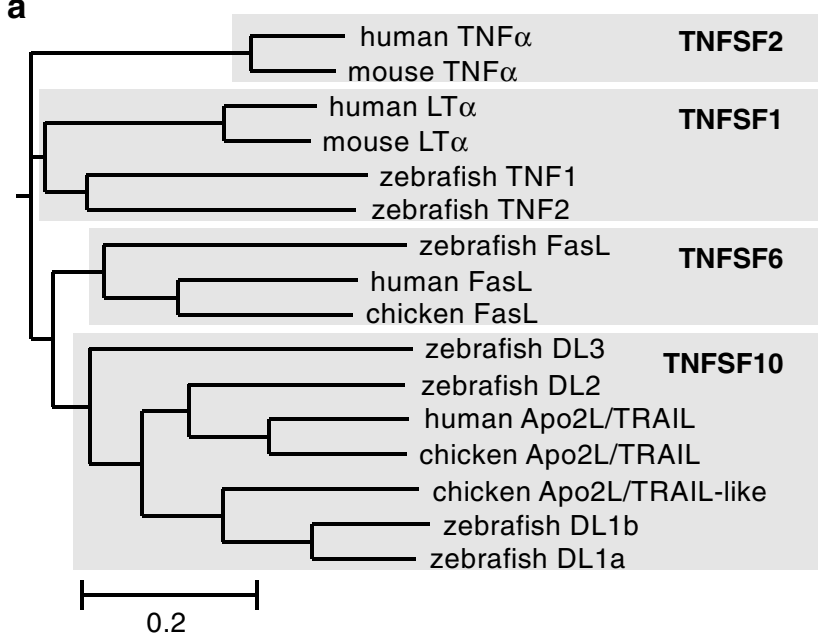

C

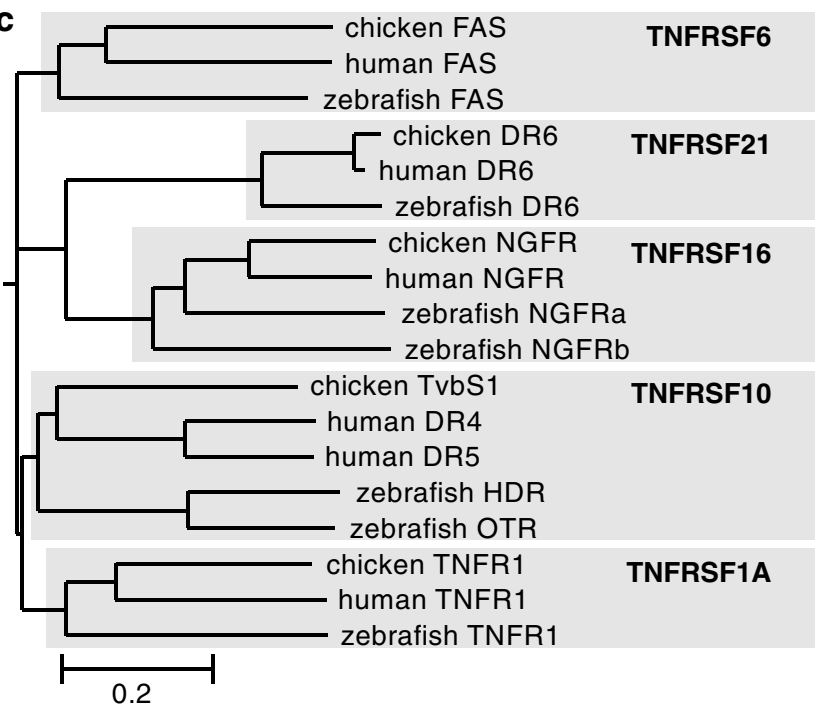

b

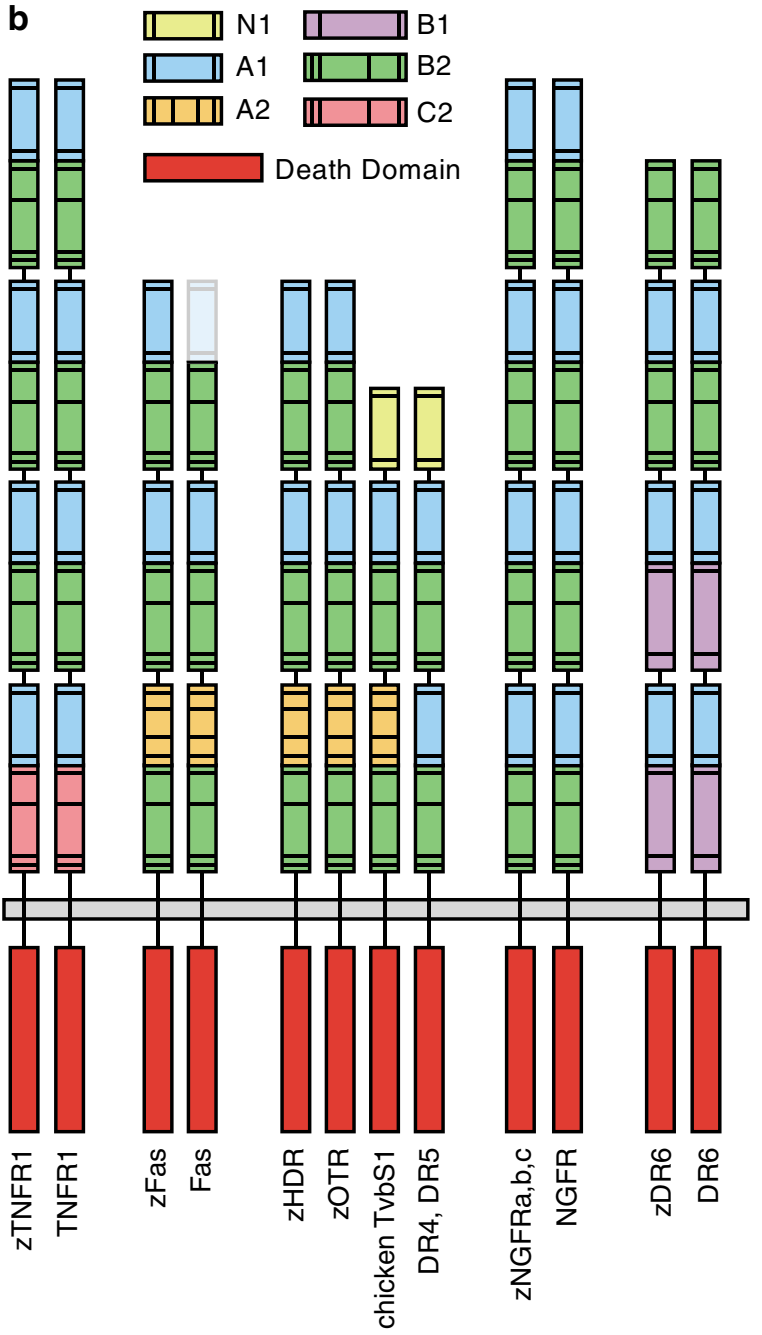

Figure 1 Structure and homology of specific TNF and TNFR superfamily members. (a) Dendrogram of TNF domains of zebrafish, mammalian, and avian ligands. (b) Schematic representation of zebrafish and mammalian DD-containing TNFRs using the domain classification system found in Bodmer et al. ${ }^{44}$ Black bars within individual modules indicate the number and relative positions of cysteine residues. The N-terminal A1 module of mammalian Fas is present in murine but not human Fas and is rendered as semitransparent. Chicken TvbS1 is included to illustrate the diversity of ECDs in the TNFRSF10 group. (c) Dendrogram of DDs of zebrafish, mammalian, and avian receptors. All sequence alignments were produced by the ClustalW algorithm 
zDL3 for Death Ligand (DL); Figure 1a). Although zDL1a was described previously, its published sequence (Accession \#AF250041) is incomplete, lacking both intracellular and transmembrane regions (full-length sequence Accession \#DQ812112). The zebrafish genome also encodes two proteins (designated zTNF1 and zTNF2), previously described as zebrafish TNF $\alpha$ orthologs based on similarity to other teleost TNF $\alpha$ genes. ${ }^{20,21}$ The genomic regions encoding zDL2 and zTNF2 in zebrafish have conserved synteny with human loci encoding Apo2L/TRAIL and TNF $\alpha$, respectively (Supplementary Table 1 and Figure 1a).

We identified eight DD-containing TNFR superfamily members in zebrafish. Six of these - zTNFR1, zFas, zDR6, zNGFRa, zNGFRb, and zNGFRc - can be matched with mammalian orthologs based on the architecture of their extracellular domains (ECDs), a dendrogram of their DDs, and conserved synteny with human counterparts (Figure $1 \mathrm{~b}$ and c; Supplementary Figure 1a). Mammalian homologs of zHDR and zOTR were more difficult to identify by sequence alone. Their ECDs resemble CD40, herpes !QJ;virus entry mediator (HVEM), and Fas while their DDs are most homologous to DR4, DR5, and avian TvbS1 (Figure 1b and c). Multiple independently amplified zOTR clones revealed that the GenBank sequence for this gene (Accession \#AAG24365) contains a missense mutation; we therefore submitted a corrected sequence (Accession \#DQ812116).

We also identified homologs of FADD, TRADD, and numerous initiator and effector caspases (Supplementary Table 1 and Figure 1b). There are at least six zebrafish caspases with protease domains more homologous to caspase-8 and -10 than to other known caspases (Supplementary Figure 1c). Both zCaspase-8a and zCaspase-8b have $\mathrm{N}$-terminal death effector domains (DEDs), but only zCaspase-8a contains the QACQG active-site motif characteristic of mammalian caspase-8 and -10 . zCaspase-8a-like is highly homologous to zCaspase-8a in the protease domain but has a truncated $\mathrm{N}$-terminus lacking DEDs. zCaspase-Xa, $-\mathrm{Xb}$, and $-\mathrm{Xc}$ are most homologous overall to mammalian caspase-8, but contain a QACRG motif and lack obvious $\mathrm{N}$-terminal DEDs. We found a single ortholog of the long splice form of c-FLIP. Like its mammalian counterpart, zFLIP is structurally similar to caspase- 8 and -10 but lacks an activesite cysteine.

Figure 2 Specific zebrafish extrinsic pathway genes induce cell death in developing zebrafish embryos. (a) Overexpression of zHDR, zOTR, zFADD $z$ Caspase-3a, and $z$ Caspase-8a in zebrafish embryos induces apoptosis. Embryos were injected with $500 \mathrm{pg}$ of the indicated mRNA and photographed at 8 h.p.f. (top panels) or fixed at 5-8 h.p.f. for antiactive caspase-3 immunohistochemistry (bottom panels). (b) Ectopic expression of zHDR, zOTR, zFas, $Z F A D D, Z T R A D D$, and caspases, but not $z F L I P$, results in embryonic mortality. Embryos were injected with $500 \mathrm{pg}$ of the indicated mRNA ( $z H D R, z O T R$, and $z$ Fas were found to be potent inducers of apoptosis and used at $200 \mathrm{pg}$ ) and embryonic mortality (defined as lysis of the yolk cell and/or blastomeres) was scored at 8 h.p.f. Significant mortality was observed subsequent to 8 h.p.f. for $z F A D D, z T R A D D$, and caspases, so these were also assessed at 24 h.p.f. $\mathrm{N}=$ number of independent experiments; $n=$ number of embryos scored. Error bars indicate standard error of the mean between experiments. (c) Overexpression of zebrafish receptors and ZFADD in human HEK-293 and HeLa cells. Cells were transfected with constructs encoding the indicated proteins and the proportion of cells remaining viable at $24 \mathrm{~h}$ was recorded

\section{Ectopic expression of $z H D R, z O T R, z F a s, z F A D D$, $z T R A D D$, and caspases induces apoptosis}

We injected zebrafish embryos with mRNAs encoding zHDR, zOTR, zFas, zDR6, or zTNFR1 to determine if these DDcontaining TNFRs can trigger apoptosis. Nearly all embryos overexpressing $z H D R, z O T R$, or zFas displayed morphological changes consistent with apoptosis and died by $8 \mathrm{~h}$ postfertilization (h.p.f.; Figure 2a and b). To confirm caspase activation, we used a monoclonal antibody that recognizes the

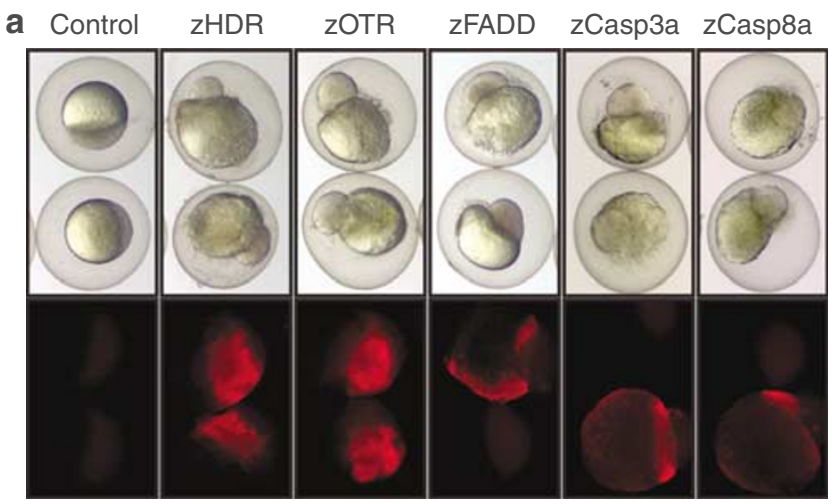

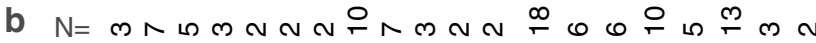

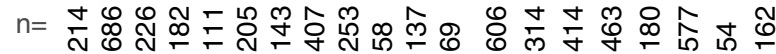

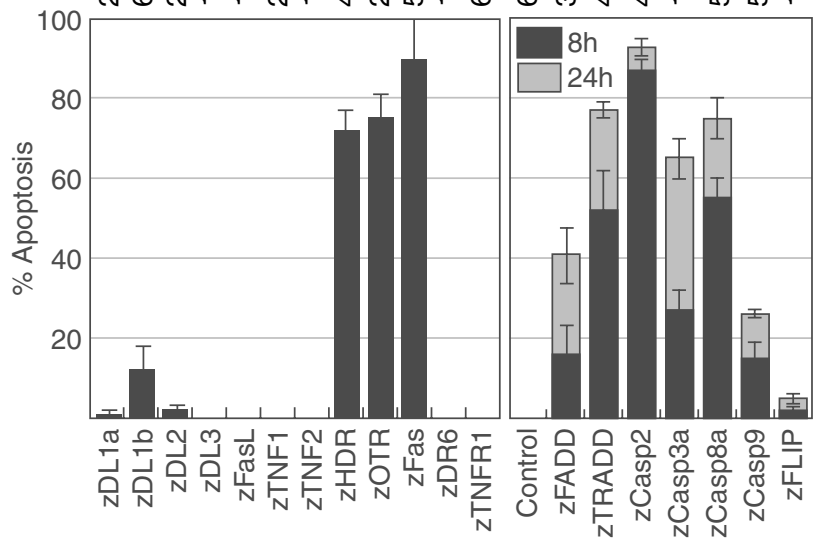

C

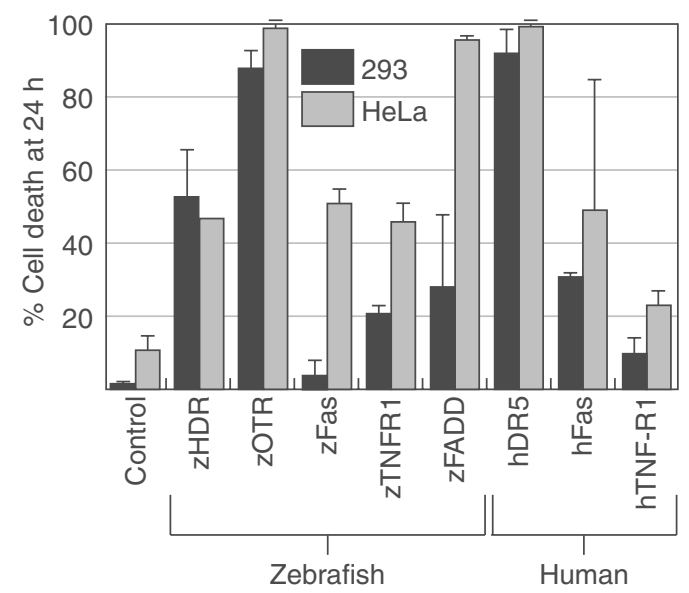


cleaved, active form of caspase-3. Immunostaining of dying embryos showed high levels of activated caspase-3 throughout the cells of the blastodisc and in the cytoplasm of the yolk cell (Figure 2a). Mock-injected embryos, or those overexpressing $z D R 6$ or $z T N F R 1$, did not exhibit apoptotic morphology or caspase-3 activation. These data correspond well with mammals, where TNFR1 and DR6 are implicated mainly in nonapoptotic responses, while Fas activation is directly linked to apoptosis. ${ }^{3}$

Injection of $z F A D D, z T R A D D$, zCaspase-2, zCaspase-3a, zCaspase-8a, and zCaspase- 9 mRNAs induced extensive apoptosis and embryonic death between 8 and 24 h.p.f. (Figure $2 \mathrm{a}$ and b). Embryos surviving beyond 24 h.p.f. displayed abnormal morphology and had numerous active caspase-3-staining cells in all tissues (not shown). In contrast, zFLIP, zDL1a, zDL2, zDL3, zFasL, zTNF1, or zTNF2 injection did not increase mortality (Figure $2 b$ ). Overexpression of $z D L 1 b$ caused a modest amount of death by 8 h.p.f. $(\sim 12 \%)$, but surviving embryos lacked the indiscriminate apoptosis triggered by injection of $z F A D D, z T R A D D$, or caspase homologs.
We also tested the activity of zebrafish receptors and zFADD upon transient overexpression in human cells. We transfected HeLa and human embryonic kidney (HEK)-293 cells with vectors encoding zHDR, zOTR, zFas, zTNFR1, or zFADD. After $24 \mathrm{~h}$, cells overexpressing zebrafish receptors or zFADD showed substantially higher levels of cell death than those transfected with an enhanced green fluorescent protein (EGFP)-expressing control vector (Figure 2c). zHDR and $z O T R$ elicited the most robust effect, inducing death in $50-100 \%$ of the transfected cells.

\section{Ectopic $z D L 1 a, z D L 1 b$, and $z D L 2$ expression induces apoptosis in notochord and hematopoietic cells}

Although ligand overexpression did not increase mortality, $z D L 1 a, z D L 1 b$, and $z D L 2$ produced striking morphological defects. Injected embryos showed greatly reduced tail lengths, flattened somites lacking a horizontal myoseptum (Figure 3a-c; Supplementary Figure 2a), and aberrant posterior vasculature (not shown). Embryos injected with

a
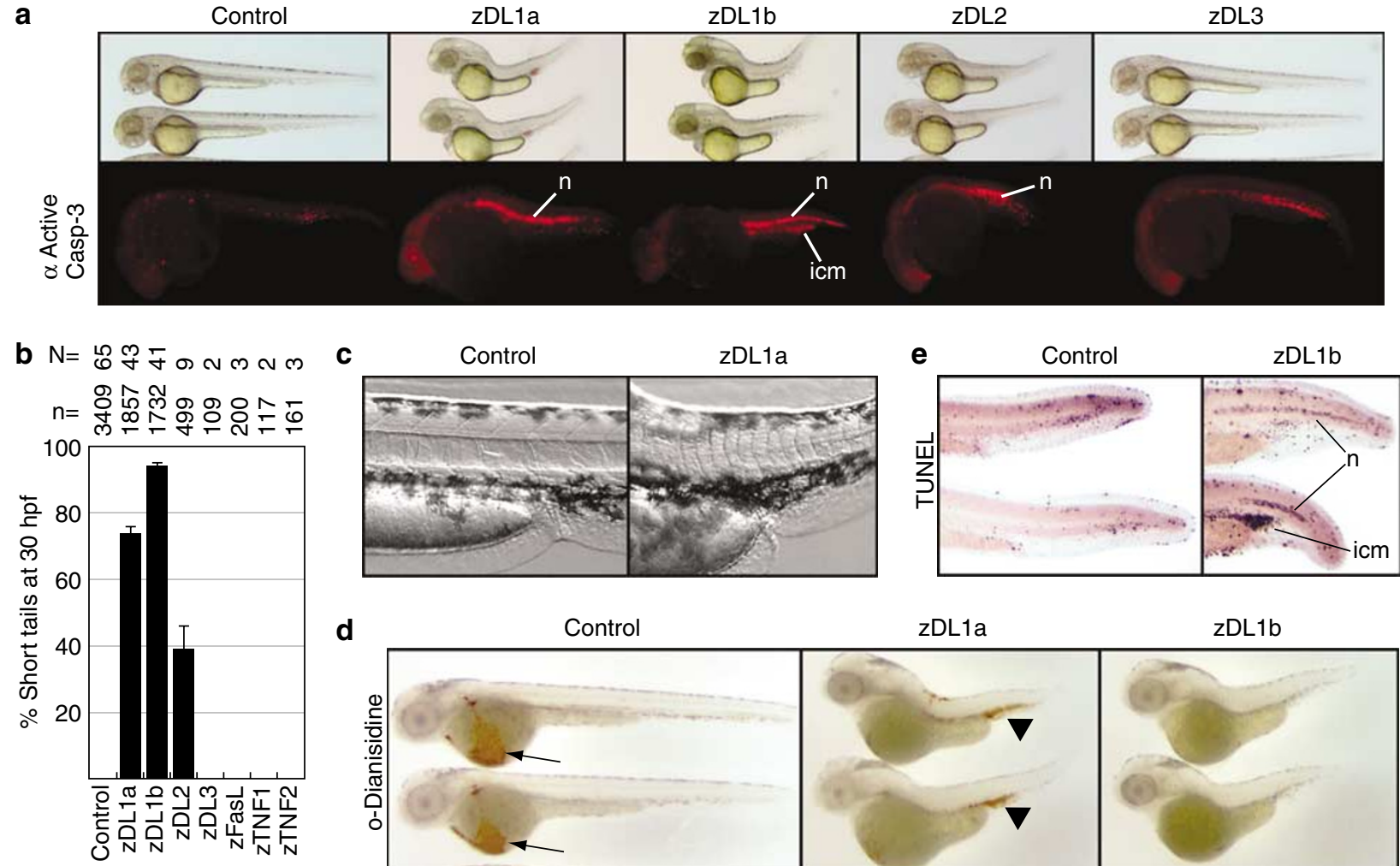

d
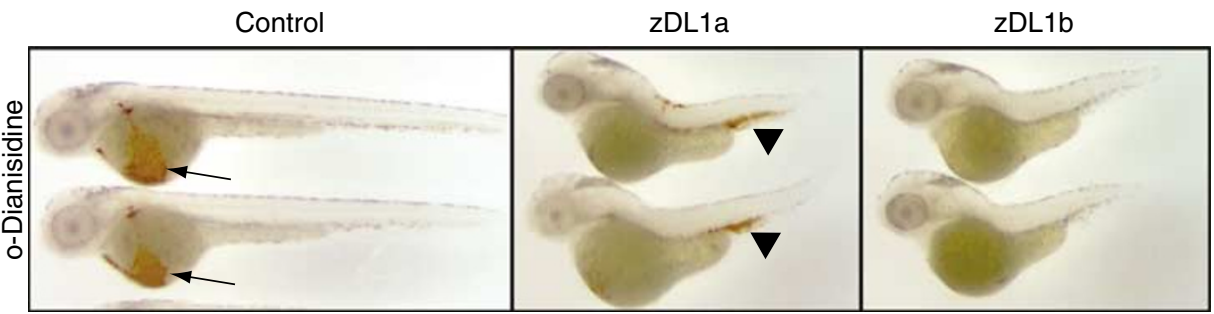

Figure $3 z D L 1 a, z D L 1 b$, and $z D L 2$ selectively kill cells of the notochord and ICM during embryogenesis. (a) Overexpression of $z D L 1 a, z D L 1 b$, and $z D L 2$ leads to 'short-tail' embryos with activated caspase-3 in notochord cells; $z D L 1 b$ also activates caspase-3 in the ICM. All embryos were injected with $500 \mathrm{pg}$ of mRNA except for $z D L 1 b$, which was found to be much more potent and used at $200 \mathrm{pg}$. Embryos were photographed at 48 h.p.f. (top panels) or fixed at 30 h.p.f. for antiactive caspase-3 immunohistochemistry (bottom panels). Abbreviations: n, notochord; icm, intermediate cell mass. (b) Only $z D L 1 a, z D L 1 b$, and zDL2 produce short-tail embryos. Embryos were injected with $500 \mathrm{pg}$ of mRNA (200 pg of $z D L 1 \mathrm{~b})$ and scored at 30 h.p.f. for tail length; only embryos with unambiguous reductions in tail length were recorded as short-tail. $\mathrm{N}=$ number of independent experiments; $n=$ number of embryos scored. Error bars indicate standard error of the mean between experiments (c) Somites in zDL1a overexpressing embryos are flattened and lack a horizontal myoseptum. Embryos were injected with 500 pg zDL1a and photographed at 48 h.p.f. (d) Mature erythrocytes pool in the perianal region of zDL1a-injected embryos (arrowheads) and are completely absent in $z D L 1 b$-injected embryos. Embryos were injected with $z D L 1 a(500 \mathrm{pg})$ or $z D L 1 b(200 \mathrm{pg})$ and whole-mount 0 -dianisidine staining for hemoglobin was carried out at 48 h.p.f. Arrows indicate circulating erythrocytes in wild-type embryos. (e) Increased numbers of TUNEL-positive cells are present in the notochord and ICM of $z D L 1 b$-injected embryos. Embryos were injected with $200 \mathrm{pg} z D L 1 b$, fixed at 30 h.p.f., and apoptotic cells were labeled by the TUNEL method. Abbreviations: $\mathrm{n}$, notochord; icm, intermediate cell mass 
zDL1a (Figure 3d) and $z D L 2$ (not shown) also exhibited pooling of erythrocytes in the perianal region. This combination of phenotypes resembles known mutants with defects in notochord formation such as floating head and no tail (nt). ${ }^{25,26}$ The notochord is a rod-like structure found in all chordate embryos that functions as a major skeletal element and an essential signaling center for tissue patterning. Injection of $z D L 3, z F a s L, z T N F 1$, or $z T N F 2$ did not cause discernible defects (Figure $3 b$ ).

Owing to the similarities between notochord mutants and ligand-injected embryos, we looked for notochord apoptosis. Injection of $z D L 1 a, z D L 1 b$, and to a lesser extent $z D L 2$, induced strong activation of caspase-3 in notochord cells (Figure 3a). We verified apoptosis using the terminal deoxynucleotidyl transferase-mediated dUTP nick end labeling (TUNEL) method, which detects apoptotic DNA fragmentation. We found numerous TUNEL-positive cells in the notochords of $z D L 1 a, z D L 1 b$, and $z D L 2$-injected embryos (Figure 3e; $z D L 1 a$ and $z D L 2$ not shown). In contrast, the notochords of mock-injected embryos contained very few TUNEL-positive cells and showed no evidence of caspase-3 activation.

$z D L 1 b$-injected embryos did not show the perianal blood pooling associated with $z D L 1 a$ and $z D L 2$ overexpression in spite of displaying an identical short-tail phenotype.
To determine whether mature erythrocytes were present, we stained embryos for hemoglobin using o-dianisidine. Embryos injected with $z D L 1 a$ had numerous erythrocytes trapped in the perianal region while those injected with $z D L 1 b$ showed virtually no o-dianisidine staining (Figure 3d). In zebrafish and other teleosts, primitive (embryonic) hematopoiesis occurs in a region of the trunk ventral to the notochord known as the intermediate cell mass (ICM). ${ }^{27}$ Activated caspase-3 staining and TUNEL revealed numerous apoptotic cells in the ICM of $z D L 1 b$-injected embryos at 30 h.p.f. (Figure $3 a$ and e).

\section{Expression of extrinsic pathway genes in the notochord and ICM}

We analyzed the zebrafish extrinsic pathway genes for notochord and ICM-specific expression patterns using whole-mount in situ hybridization (ISH) (Figure 4a-I). At 8 h.p.f. (75\% epiboly), we detected $Z O T R$ and $Z F A D D$ in the embryonic shield, a dorsal population of cells which gives rise to the notochord (Figure 4d and j). By 16h.p.f. (14-somite stage), both transcripts, as well as $z H D R$ (Figure 4c), were found throughout the notochord. We confirmed notochordspecific expression at this stage in sectioned embryos (Figure $4 \mathrm{e}$ for $z H D R$ ). By 30 h.p.f., all three transcripts were
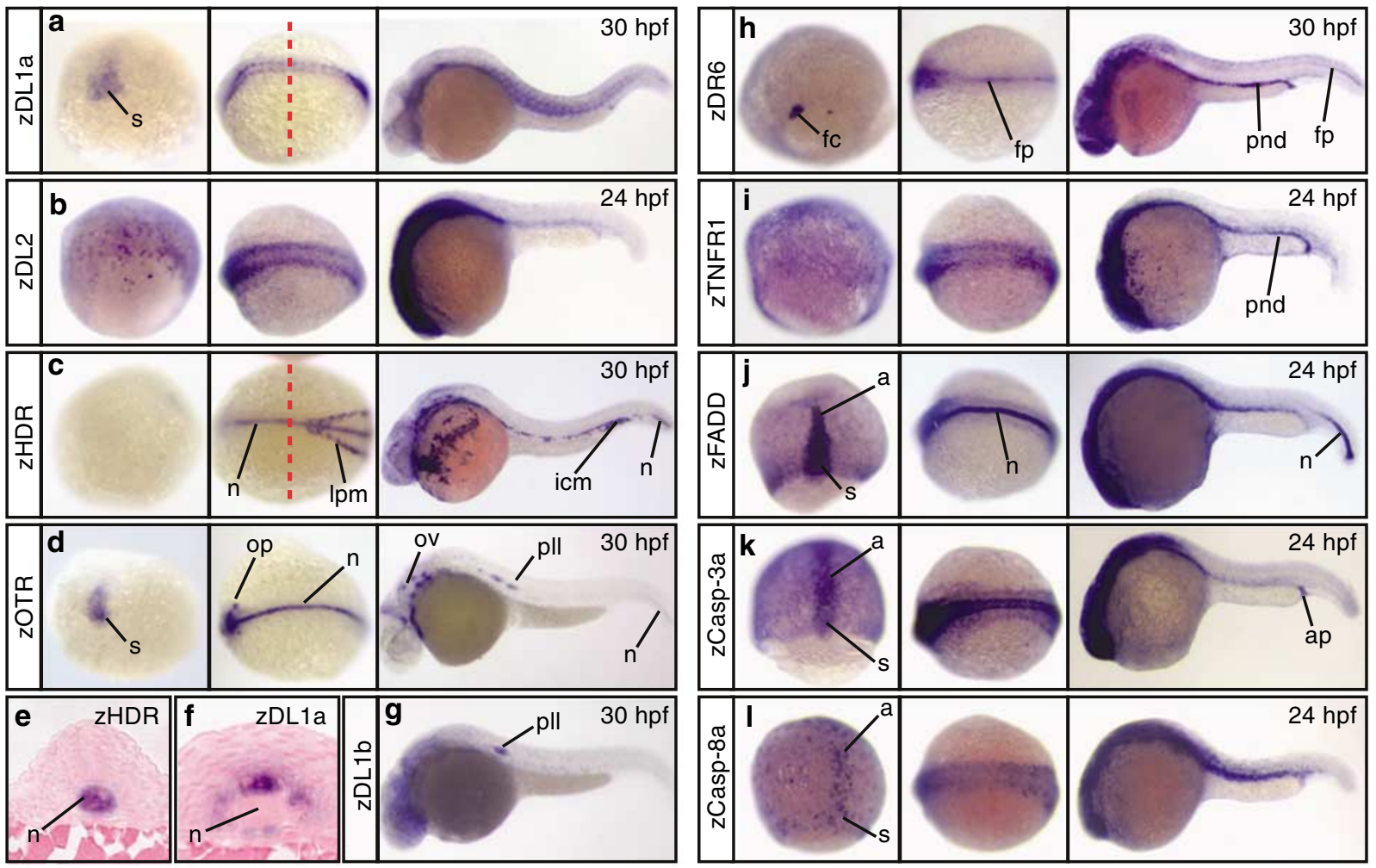

Figure 4 Embryonic gene expression. (a-d; g-l) Whole-mount ISH staining (blue) for the indicated genes at 8 h.p.f. (left), 16 h.p.f. (center), and 24-30 h.p.f. (right). 16 h.p.f. embryos are shown in dorsal view and 24-30 h.p.f. embryos are shown in lateral view. Anterior is to the left. The following abbreviations are used to indicate sites of expression: a, embryonic axis; ap, anal pore; fc, forerunner cells; fp, floorplate; icm, intermediate cell mass; Ipm, lateral plate mesoderm; $n$, notochord; op, otic placode; ov, otic vesicle; pll, posterior lateral line; pnd, pronephric duct; s, shield. (b) Staining has been allowed to develop overnight to visualize low levels of transcript throughout the body. Similar nonlocalized staining was noted for $z D L 1 a$ and $z D L 1 b$, but not a control probe, following overnight incubation. (e-f) Transverse sections illustrating notochord-associated expression (purple) of $z H D R$ and $z D L 1 a$ at 16 h.p.f. Sections have been counterstained with Nuclear Fast Red. Dashed red lines in (c) and (a) indicate plane of section in (e) and (f), respectively 
limited to the posterior notochord. This dynamic expression pattern is highly reminiscent of classic notochord markers such as sonic hedgehog (shh) and $n t l . z D L 1 a$ was the only ligand associated specifically with the notochord (Figure 4a). We detected expression in the embryonic shield at 8 h.p.f. and in bilateral, segmented stripes flanking the notochord at 16 h.p.f. Sectioning revealed strong $z D L 1 a$ expression in cell populations directly abutting the notochord (the floorplate of the neural tube, the medial cells of the somites, and the underlying endoderm), but not in the notochord itself (Figure 4f). zHDR was the only extrinsic pathway gene expressed specifically in the ICM (Figure 4c). Consistent with previous reports, ${ }^{24}$ we first observed $z H D R$ transcripts at 16 h.p.f. in the posterior lateral plate mesoderm, which gives rise to the ICM. By 30 h.p.f., $z H D R$ was strongly expressed in both the ICM and circulating erythroid cells.

\section{Additional sites of embryonic expression}

We observed embryonic expression of zebrafish extrinsic pathway genes in a number of tissues in addition to the notochord and ICM (Figure 4a-I). Only zDL3, zFasL, zTNF1, $z T N F 2$, and $z F a s$ were not seen by whole-mount ISH during the first $48 \mathrm{~h}$ of development (not shown), although in some cases these transcripts were detected by RT-PCR (Supplementary Figure 3 ). We found strong expression of $z D L 1 b$, $z D L 2$, and $z O T R$ in cells of the lateral line (Figure $5 a$ ), a mechanosensory system used by fish and amphibians to detect water displacement. ${ }^{28}$ The lateral line is composed of individual sense organs called neuromasts that originate in the otic region and become spread along the body axis by long-range migration. ${ }^{29}$ We detected $z O T R$ in the otic placode at 16 h.p.f., and both $z O T R$ and $z D L 1 b$ in the migrating posterior lateral line (PLL) primordia at 30 h.p.f. (Figure $4 \mathrm{~d}$ and g). By 48 h.p.f., $z O T R, z D L 1 b$, and $z D L 2$ were observed in all 7-8 PLL neuromasts and all cranial lateral line neuromasts (Figure 5a). Sectioning confirmed neuromast expression of $z O T R$ and revealed high levels of $z D L 1 a, z D L 1 b, z D L 2$, $z O T R$, and $z T N F R 1$ in the otic vesicles at 48 h.p.f. (Figure $5 \mathrm{~b}$; $z D L 1 b$ and $z D L 2$ not shown). Both the otic vesicles and the lateral line neuromasts employ hair cells similar to those of the mammalian inner ear to detect stimuli. ${ }^{30}$ In light of the observed expression patterns, it is interesting to note that zebrafish hair cells undergo constant, apoptosis-mediated turnover. $^{31}$

We detected expression of several zebrafish TNF superfamily ligands and receptors in the developing central nervous a

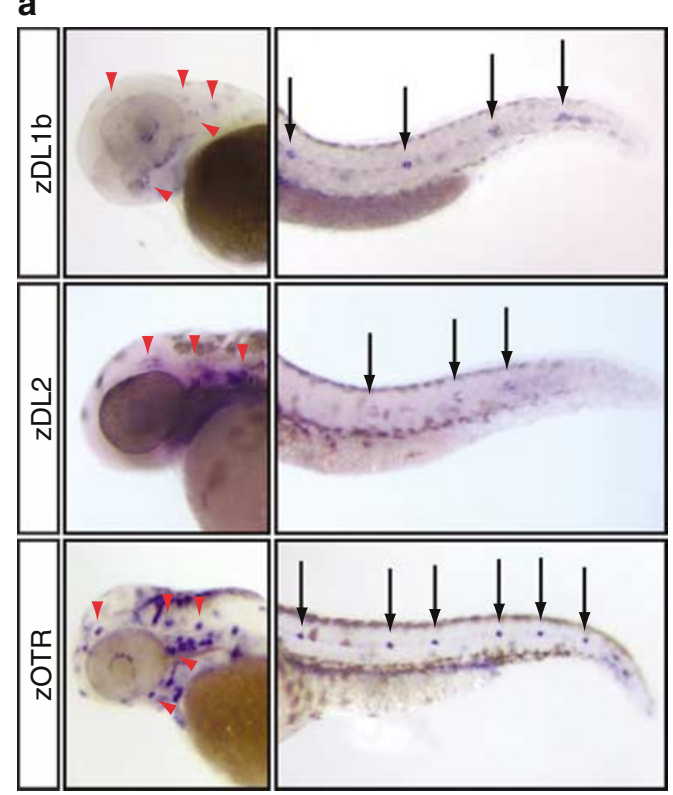

b

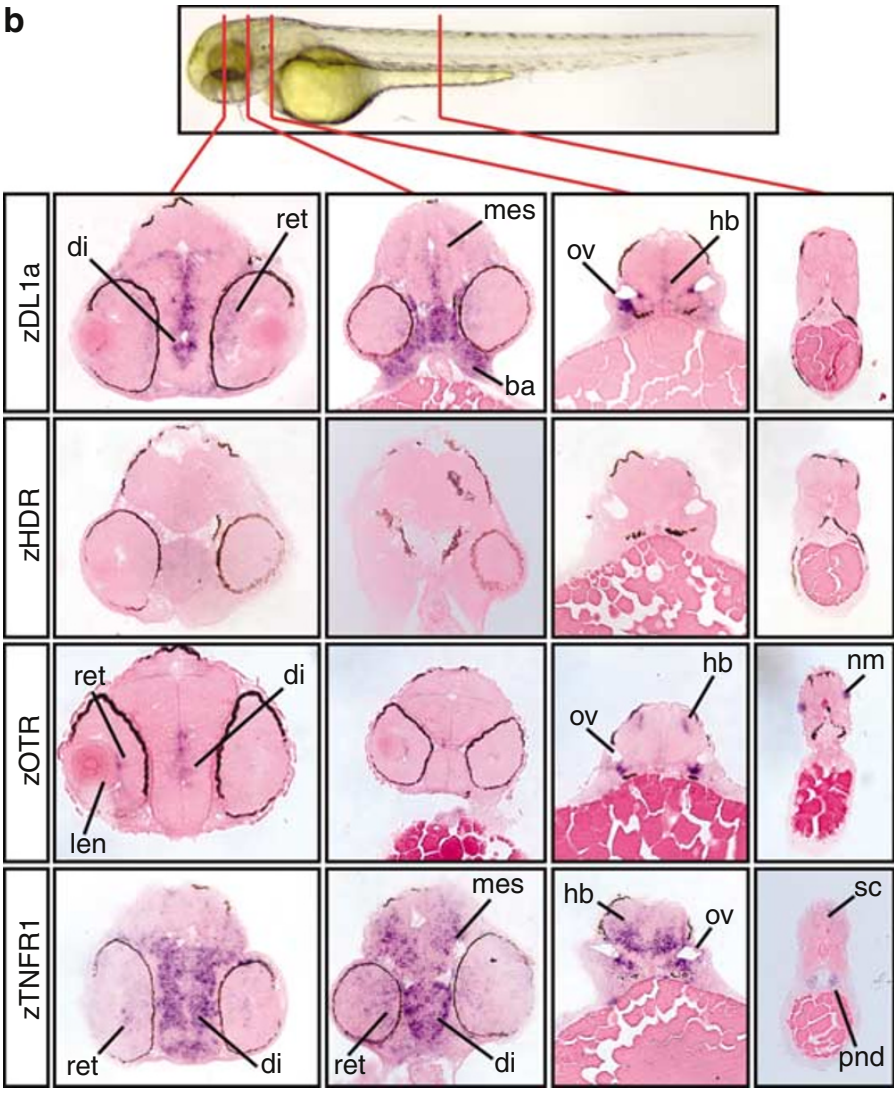

Figure 5 Expression in the developing lateral line and CNS. (a) 48 h.p.f. embryos stained by whole-mount ISH (blue) for $z D L 1 b, z D L 2$, and $z O T R$. Arrows indicate expression in posterior lateral line neuromasts and red arrowheads indicate expression in cranial lateral line neuromasts. (b) Transverse sections through $48 \mathrm{~h} . p . f$. embryos stained by whole-mount ISH (purple) for zDL1a, zHDR, zOTR, and zTNFR1. zHDR is not detected in the body at this stage and serves as a background control. Sections have been counterstained with Nuclear Fast Red. The following abbreviations are used to indicate sites of expression: ba, branchial arch; di, diencephalon; hb, hindbrain; len, lens; mes, mesencephalon; nm, neuromast; ov, otic vesicle; pnd, pronephric duct; ret, neural retina; sc, spinal chord 
system (CNS). The earliest of these is $z D R 6$, which was expressed in the floorplate of the neural tube at $16 \mathrm{~h}$.p.f. and in the forebrain, midbrain, and hindbrain at 30 h.p.f. (Figure 4h). Other genes, including $z D L 1 a, z D L 1 b$, $z D L 2, z O T R$, and $z T N F R 1$, were expressed in the developing brain by 24-30 h.p.f. (Figure 4a, b, d, g, and i). We confirmed localization of TNF and TNFR transcripts to specific regions of the CNS in sectioned embryos (Figure 5b). For example, $z D L 1 a$ was localized to the ventricular surface of the diencephalon, mesencephalon, and hindbrain, while zTNFR1 was more broadly expressed throughout these regions as well as in the spinal cord. We also noted expression in the developing eye, with zOTR being localized to the boundary between lens and retina, while $z D L 1 a$ and $z T N F R 1$ were expressed throughout the neural retina.

\section{zDL1a, zDL1b, and zDL2 signal through zHDR and zOTR}

The ability of zebrafish Apo2L/TRAIL homologs to induce apoptosis specifically in embryonic tissues expressing $z H D R$ and $z O T R$ suggested that these proteins may represent ligand-receptor pairs. To test this, we generated soluble receptor constructs in which ECDs were fused to the $\mathrm{Fc}$ region of immunoglobulin $\mathrm{G} 1$ and soluble ligands that were tagged at the N-terminus with the FLAG epitope. We were able to express FLAG-tagged zDL1a, zDL1b, zDL2, and zTNF1, as well as Fc-tagged zHDR, zOTR, and zTNFR1, in HEK-293 cells. In co-immunoprecipitation studies (Figure 6a), zDL1a bound both zHDR-Fc and zOTR-Fc, with apparent preference for zOTR. zDL2 also bound both receptors, although less strongly than an equivalent amount of zDL1a. In contrast, zDL1b selectively bound zHDR-Fc and not zOTR-Fc. Neither zDL1a, zDL1b, nor zDL2 interacted with zTNFR1-Fc, while zTNF1 did, confirming that binding was specific.

We analyzed these interactions in vivo using antisense morpholino oligonucleotides (MOs) targeting $\mathrm{zHDR}$ (zHDR$\mathrm{MO}$ ) and $z O T R$ ( $z O T R-\mathrm{MO}$ ). Both $\mathrm{MOs}$ rescued overexpression of their respective mRNAs (Supplementary Figure $2 b$ ) and neither had nonspecific toxicity at doses as high as $10 \mathrm{ng}$ (not shown). Approximately, $70 \%$ of embryos coinjected with zDL1a and a control MO developed short tails by 30 h.p.f. Coinjection of $z H D R-\mathrm{MO}$ did not diminish this frequency, but coinjection of $z O T R-\mathrm{MO}$ reduced the number of short-tailed embryos to $<10 \%$ (Figure $6 \mathrm{~b}$ ). $z D L 1 b$ showed the reciprocal receptor specificity: $z H D R-M O$ almost completely blocked $z D L 1 b$-induced apoptosis in the notochord and ICM while zOTR-MO had no effect (Figure $6 \mathrm{~b}$ and c; Supplementary Figure 2a). A dominant-negative (DN) version of zHDR (zHDR-DN) lacking the cytoplasmic DD also inhibited the action of $z D L 1 b$ (not shown). Similar to $z D L 1 a$, apoptosis induced by $z D L 2$ was blocked by $z O T R-M O$ but not by $z H D R$ $\mathrm{MO}$ (Figure 6b). Thus, zDL1a and zDL2 signal in vivo through zOTR, while zDL1b signals through zHDR. This is consistent with the observation that all three ligands trigger apoptosis in the notochord (which expresses both $z H D R$ and $z O T R$ ), while only $z D L 1 b$ induces apoptosis in the ICM (which expresses $z H D R)$.

\section{$z D L 1 a$ and $z D L 1 b$ require $z F A D D$ and $z$ Caspase-8- like proteases, but not $z T R A D D$, to induce apoptosis}

We assessed the importance of zFADD, zTRADD, and caspase-8-like proteases for apoptosis induction by Apo2L/TRAIL homologs. We inhibited $Z F A D D$ with a spliceblocking $\mathrm{MO}(z F A D D-\mathrm{MO})$ that prevents excision of the large intron between the first and second exons. Coinjection of $z F A D D-\mathrm{MO}$ with either $z D L 1 a$ or $z D L 1 b$ significantly attenuated the short-tail phenotype (Figure $6 \mathrm{~d}$; Supplementary Figure 2a). We used a standard $\mathrm{MO}$, targeting the start codon, to inhibit $z T R A D D$ ( $z T R A D D-M O)$. Although coinjection $z T R A D D-\mathrm{MO}$ fully rescued embryonic mortality arising from $z T R A D D$ overexpression (Supplementary Figure $2 b)$, it failed to inhibit $z D L 1 a$ or $z D L 1 b$-induced apoptosis (Figure 6d). The presence of multiple zebrafish caspases with protease-domain homology to caspase-8 and -10 (Supplementary Figure 1c) precluded MO knockdown. Instead, we used $z F L I P$ and the antiapoptotic cowpox virus serpin cytokine response modifier A $(\mathrm{Crm} A)$. In mammals, c-FLIP interferes with DISC recruitment and activation of caspase$8,^{32}$ while CrmA inhibits caspase- $8,{ }^{33}$ caspase- $10,{ }^{34}$ and caspase $-1^{35}$ (the latter is involved in inflammation rather than apoptosis). Coinjection of $z F L I P$ or $\operatorname{CrmA}$ significantly reduced $z$ Caspase-8a-mediated embryonic mortality while having no effect on apoptosis induced by zCaspase-2 or the proapoptotic BH3-only protein zNoxa (Supplementary Figure 4; zFLIP not shown). This confirmed that both proteins function similarly in zebrafish and mammals. Coinjection of $\mathrm{CrmA}$ with $\mathrm{zHDR}$ or $z O T R$ blocked receptor-induced embryonic mortality (Supplementary Figure 4). Moreover, coinjection of $z F L I P$ or $C r m A$ with $z D L 1 a$ or $z D L 1 b$ substantially reduced the incidence of the short-tail phonotype and, with $z D L 1 b$, restored circulating erythrocytes (Figure $6 \mathrm{c}$ and d; Supplementary Figure 2a). Hence, $z D L 1 a$ and $z D L 1 b$ induced apoptosis in zebrafish requires both $z F A D D$ and caspase-8-like enzymes but not zTRADD.

\section{Zebrafish development proceeds normally without the extrinsic apoptosis pathway}

To determine the importance of the extrinsic apoptosis pathway in zebrafish embryogenesis, we injected embryos with $z H D R-M O, z O T R-M O, \quad z F A D D-M O$, or with mRNA encoding zHDR-DN or CrmA. In all cases, we found no gross morphological abnormalities during the first week of development. This was true even for simultaneous inhibition of zHDR and zOTR (Supplementary Figure 5a). There were no apparent defects in expression of tissue-specific markers for hemangioblasts, vasculature, notochord, floorplate, spinal cord neurons, neuromasts, the pronephric duct, or the myoseptum (not shown). There was also no alteration in tail length, confirming normal notochord development (Supplementary Figure 5b). Circulation, heart looping, and overall cardiac morphology appeared normal at 48 h.p.f. and o-dianisidine staining confirmed that fully differentiated erythrocytes were present. Thus, while the extrinsic pathway is operative in the zebrafish embryo, it is not crucial for normal development. 
a

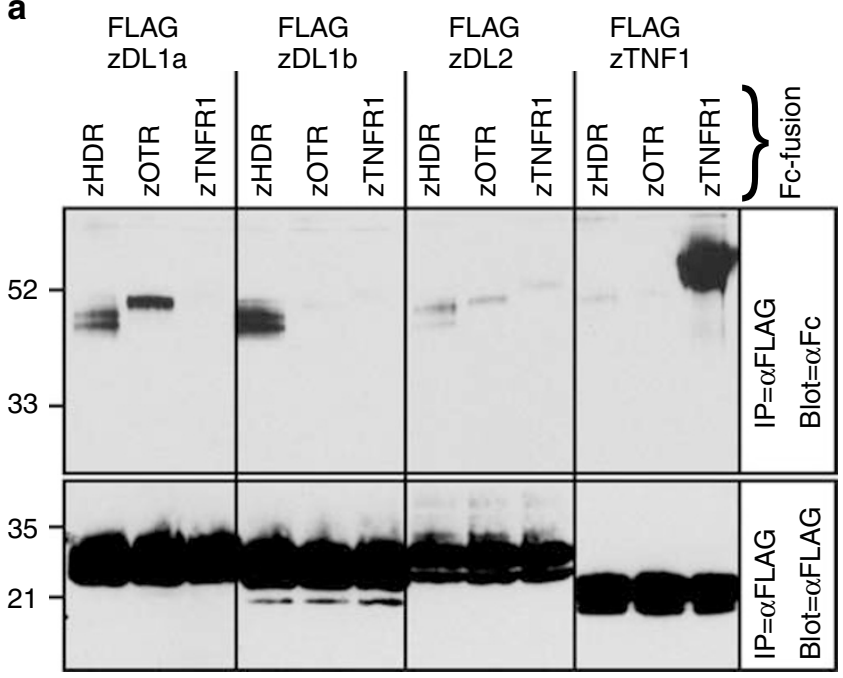

C
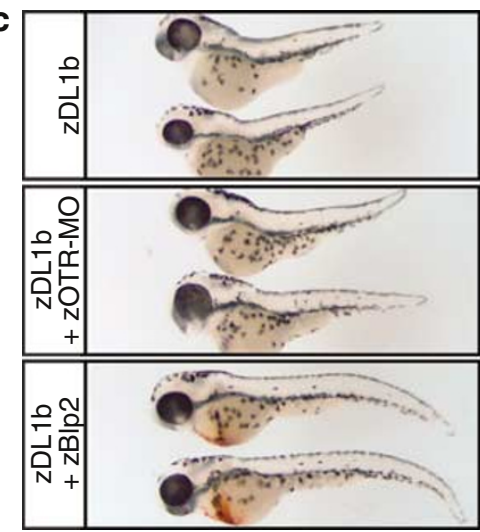
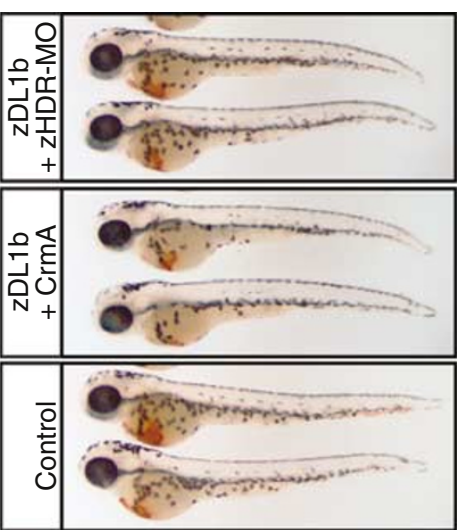

b

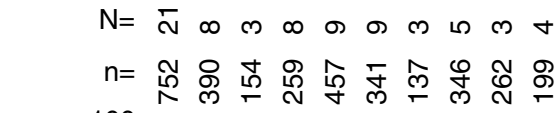

d

$$
\begin{aligned}
& \mathrm{N}=\text { ㅇ எ }
\end{aligned}
$$

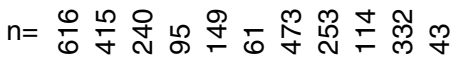

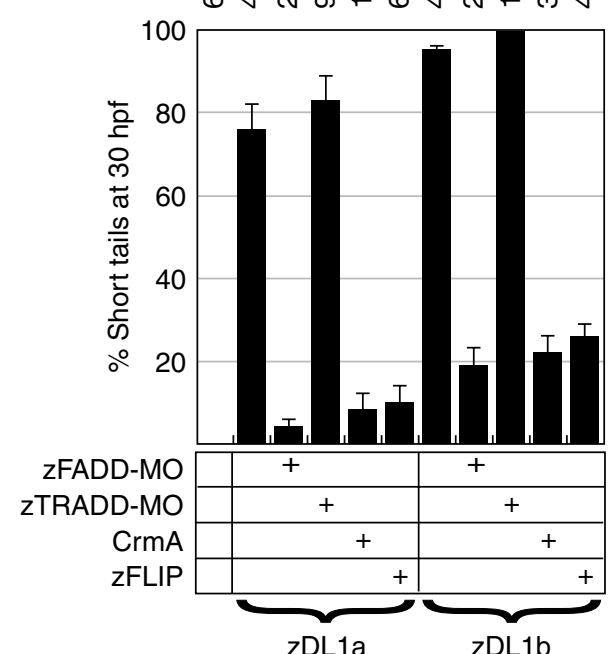

Figure 6 Characterization of the extrinsic apoptosis pathway in zebrafish. (a) Zebrafish Apo2L/TRAIL homologs directly and selectively bind to zHDR and zOTR. Soluble FLAG-tagged ligands and receptor-Fc fusion proteins were expressed in HEK-293 cells. Conditioned media from ligand and receptor-expressing cells were mixed and immunoprecipitated using anti-FLAG M2 agarose beads. The Fc region of co-precipitating receptors was detected using an anti-human IgG-specific antibody. (b) Knockdown of zHDR prevents $z D L 1 b$-mediated notochord apoptosis while knockdown of zOTR prevents zDL1a and zDL2-mediated notochord apoptosis. Embryos were injected with $500 \mathrm{pg}$ of $z D L 1 a$ or $z D L 2$ or $200 \mathrm{pg} z D L 1 b$. Five nanograms of $z H D R$-MO or $z O T R$-MO were coinjected as indicated. Only embryos with unambiguous reductions in tail length at 30 h.p.f. were scored as short-tail. (b, d) $N=$ number of independent experiments; $n=$ number of embryos scored. Error bars indicate standard error of the mean between experiments. (c) zDL1b-mediated apoptosis can be rescued by $z H D R-M O, C r m A$, and zB/p2, but not by $z O T R$-MO. Embryos were coinjected with $z D L 1 b(200 \mathrm{pg})$ and $z H D R-M O(5 \mathrm{ng}), z O T R-M O(5 \mathrm{ng}), \mathrm{CrmA}(500 \mathrm{pg})$, or zBlp2 $(500 \mathrm{pg})$ as indicated. Whole-mount 0 -dianisidine staining for hemoglobin was carried out at 48 h.p.f. (d) $z D L 1 a$ and $z D L 1 b$-mediated notochord apoptosis can be inhibited by $z F L I P$ or $C r m A$ or by knockdown of zFADD. Embryos were injected with $z D L 1 a(500 \mathrm{pg})$ or $z D L 1 b(200 \mathrm{pg})$. zFADD-MO $(5 \mathrm{ng}), z T R A D D-M O(5 \mathrm{ng})$, CrmA $(500 \mathrm{pg})$, or zFLIP (500 pg) were coinjected as indicated. Only embryos with unambiguous reductions in tail length at 30 h.p.f. were scored as short-tail

\section{Ligand-induced apoptosis in the notochord and ICM requires both the extrinsic and intrinsic pathways}

In some mammalian cell types, ligand-induced apoptosis requires amplification of the extrinsic signal by the intrinsic pathway, ${ }^{36}$ which can be blocked experimentally by overexpression of antiapoptotic members of the $\mathrm{Bcl}-2$ gene family. Coinjection of $z B / p 1$ (a homolog of $\mathrm{Bcl}-\mathrm{x}_{\mathrm{L}}{ }^{37}$ ) with $z H D R$ or
zOTR substantially reduced embryonic mortality at 8 h.p.f. (Figure $7 a$ ). Coinjection of $z B / p 1$ also protected from death induced by enforced expression of $z$ Caspase-8a, albeit only partially. An incomplete rescue is not surprising, given that high levels of activated caspase-8 in mammalian cells circumvent the requirement for the intrinsic pathway. Coinjection of $z B l p 1$ or $z B / p 2$ (a homolog of $B c l-2^{38}$ ) with $z D L 1 a$ or $z D L 1 b$ markedly attenuated the short-tail phenotype and prevented notochord and ICM apoptosis (Figures 6c, 7a and 
b; Supplementary Figure 2a). Similarly, MO-mediated inhibition of zebrafish $\mathrm{Mcl}-1$ homologs sensitized normally resistant embryonic tissues to $z D L 1 b$-induced apoptosis (Kratz et al., accompanying manuscript). These results suggest that the identified zebrafish ligands require crosstalk between the extrinsic and intrinsic pathways to trigger apoptosis in embryonic tissues.

\section{$z D L 1 b$-induced ICM apoptosis is limited to the erythroid lineage}

The ICM is the functional equivalent of the mammalian yolk sac blood islands and contains vascular, erythroid, and myeloid precursors at various stages of differentiation. ${ }^{39}$ To
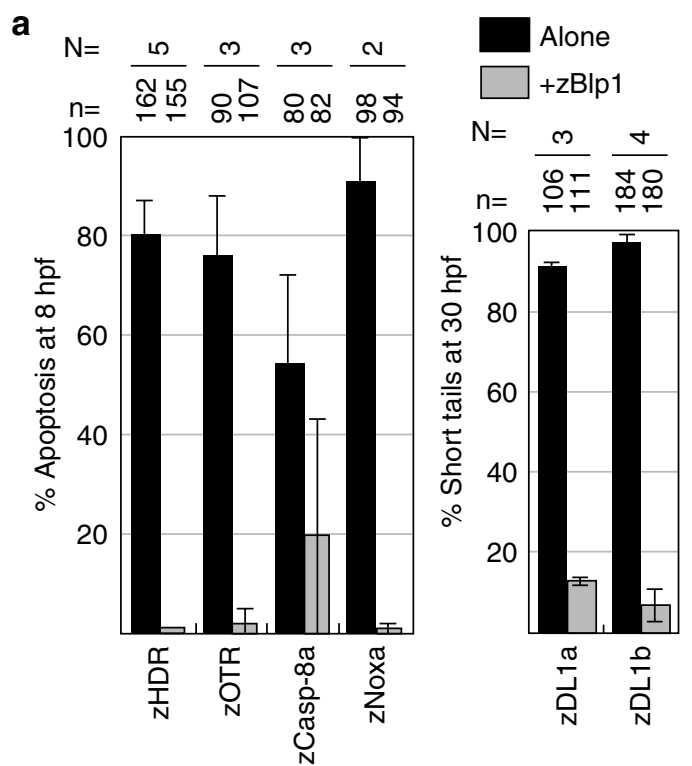

b

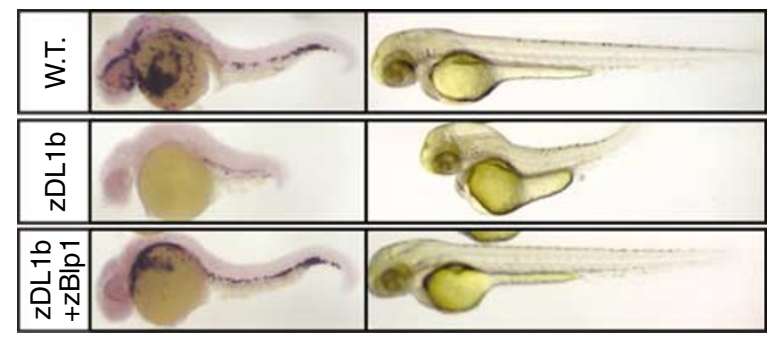

Figure 7 Zebrafish ligand-induced apoptosis requires the intrinsic apoptosis pathway. (a) The zebrafish Bcl- $x_{L}$ ortholog zBlp1 inhibits apoptosis induced by components of the extrinsic pathway. Embryos were injected with indicated mRNAs either alone or in conjunction with of $z B / p 1$ (500 pg). Embryonic death induced by $z H D R(300 \mathrm{pg}), z O T R(300 \mathrm{pg}), z$ Caspase-8a $(300 \mathrm{pg})$, or $z$ Noxa $(100 \mathrm{pg})$ was scored at 8 h.p.f. (left panel) and the short-tail phenotype induced by $z D L 1 a(500 \mathrm{pg})$ or $z D L 1 b(200 \mathrm{pg})$ was scored at 30 h.p.f. (right panel). Only embryos with unambiguous reductions in tail length were recorded as short-tail. $\mathrm{N}=$ number of independent experiments; $n=$ number of embryos scored. Error bars indicate standard error of the mean between experiments. (b) Zebrafish notochord cells and erythroblasts require the intrinsic apoptosis pathway for ligand-mediated killing. Embryos were injected with $z D L 1 b(200 \mathrm{pg})$ alone or in combination with $z B / p 1(500 \mathrm{pg})$. Embryos were photographed at 48 h.p.f. (right panels) or fixed at 30 h.p.f. for whole-mount ISH to detect the erythrocyte marker globin $\alpha e 1$ (left panels) determine which ICM cell types respond to $z D L 1 b$, we performed whole-mount ISH and quantitative PCR (QPCR) analysis for an array of hematopoietic and vascular markers (Figure 8a). Injection of $z D L 1 b$ led to a nearly complete loss of mature erythrocytes expressing embryonic $\alpha$ - and $\beta$-globin subunits (Figure $8 \mathrm{~b}$ and $\mathrm{c}$ ). In contrast, $z D L 1 b$ overexpression had no effect on $s c l, f l k 1$, and gata2, which are expressed much earlier in the hematopoietic program and associated with hemangioblasts, hematopoietic stem cells, and angioblasts (Figure $8 \mathrm{~b}$ ). The first unambiguous marker of erythroid commitment is the transcription factor gata $1 .{ }^{40}$ Whole-mount ISH and QPCR analysis on $z D L 1 b$-injected embryos revealed complete loss of gata1 (Figure $8 \mathrm{~b}$ and $\mathrm{c}$ ), demonstrating that hematopoietic cells become sensitive to $z D L 1 b$-mediated apoptosis only as erythroid differentiation begins. $z D L 1 b$ did not affect expression of the myeloid-specific markers draculin (not shown), pu.1 (Figure 8b), or L-plastin (Figure 8c) in either the ICM or the rostral blood island (RBI). Hence, even among cells that adopt a hematopoietic fate, only erythroid precursors respond to $z D L 1 b$ by apoptosis.

\section{Discussion}

The cell-extrinsic apoptosis pathway is well characterized in mammals, but has remained poorly defined in other vertebrates. Drosophila studies show that the role of the TNF superfamily in apoptosis predates the chordate lineage. However, TNF superfamily ligands use different pathways to activate initiator caspases in fruit flies versus mammals. ${ }^{8}$ By systematically characterizing the extrinsic pathway in zebrafish, we have shown that its various basic components are highly conserved throughout vertebrate evolution (Figure 9). As in mammals, apoptosis induction through the extrinsic pathway requires FADD and at least one caspase-8-like protease. Like certain mammalian cell types, ligand-induced apoptosis in the zebrafish notochord and ICM requires crosstalk between the extrinsic and intrinsic pathways. These similarities between distant species suggest that the extrinsic apoptosis pathway coopted FADD and caspase-8 early in vertebrate evolution.

Of the four zebrafish Apo2L/TRAIL homologs, zDL2 is most structurally similar to mammalian and avian Apo2L/TRAIL, while zDL1a and zDL1b are most homologous to a chicken 'Apo2L/TRAIL-like' protein (Accession \#AAL23702) (Figure 1a). Chicken Apo2L/TRAIL-like, zDL1a, and zDL1b share two additional ECD cysteine residues (corresponding to L147 and Y209 in human Apo2L/TRAIL) that are not found in other homologs. ${ }^{41}$ We propose that the ancestral gene was duplicated before the divergence of ray-finned and lobefinned fishes $\sim 400$ million years ago, giving rise to $D L 1$ (Apo2L/TRAIL-like) and DL2 (Apo2L/TRAIL) variants. A further chromosomal doubling event within the ray-finned fish lineage ${ }^{42}$ presumably duplicated the $D L 1$ gene locus, producing $D L 1 a$ and $D L 1 b$. Modern teleosts and birds have retained both $D L 1$ and $D L 2$, while the $D L 1$ variant is absent in at least some mammals including humans and mice.

We have established that three of the four zebrafish Apo2L/ TRAIL homologs interact with zHDR and zOTR. This finding is somewhat surprising, as the ECDs of both receptors differ 
a
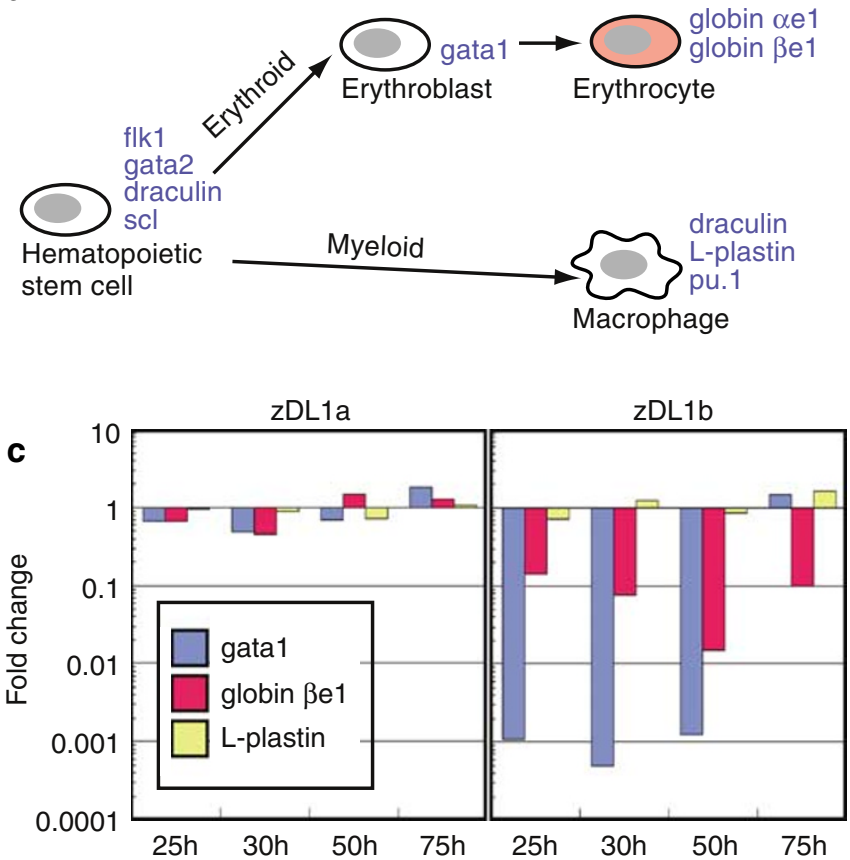

b

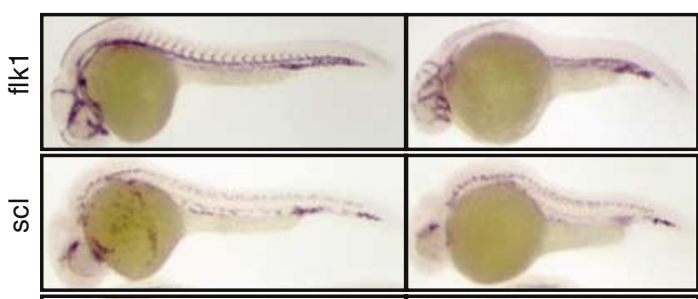

ণ্ণ

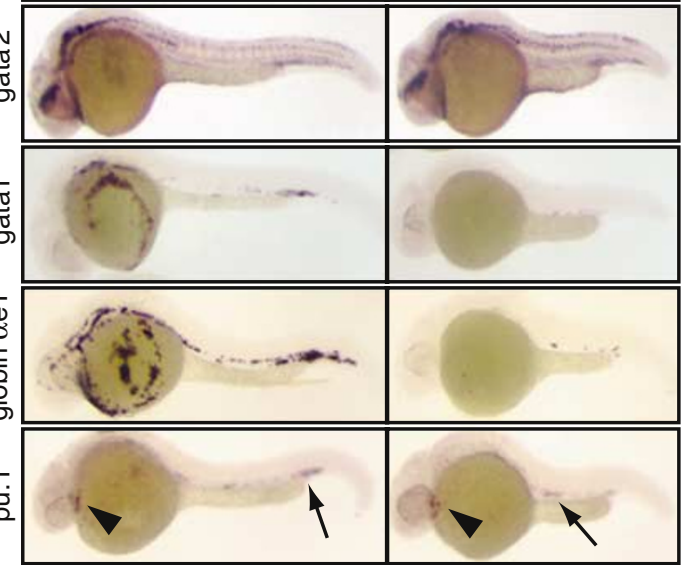

Figure $8 \quad z D L 1 b$ selectively induces erythroblast apoptosis in the ICM. (a) Diagram of primitive hematopoiesis in zebrafish, illustrating characteristic markers. (b) $z D L 1 b$ overexpression leads to loss of marker genes for erythroblasts and mature erythrocytes. Embryos were injected with $200 \mathrm{pg}$ of $z D L 1 \mathrm{~b}$ and fixed at $30 \mathrm{~h}$.p.f. for wholemount ISH. zDL1b-injected embryos show ICM expression of early hematopoietic markers (flk1, scl, gata2) but not markers of more mature erythrocytes (gata1, globin $\alpha$ e1). The myeloid-specific transcription factor pu. 1 is also unaffected. Arrowhead = RBI expression; arrow = ICM expression (c) QPCR analysis of relative gata1, globin $\beta e 1$, and $L$-plastin mRNA levels in $z D L 1 a$ and $z D L 1 b$-injected embryos. Embryos were injected with $z D L 1 a(500 \mathrm{pg})$ or $z D L 1 b(200 \mathrm{pg})$ and total mRNA was isolated at the indicated times. mRNA levels are expressed as the fold difference between ligand-injected and control embryos (control embryos were injected with $500 \mathrm{pg} E G F P$ mRNA and have a value of 1)

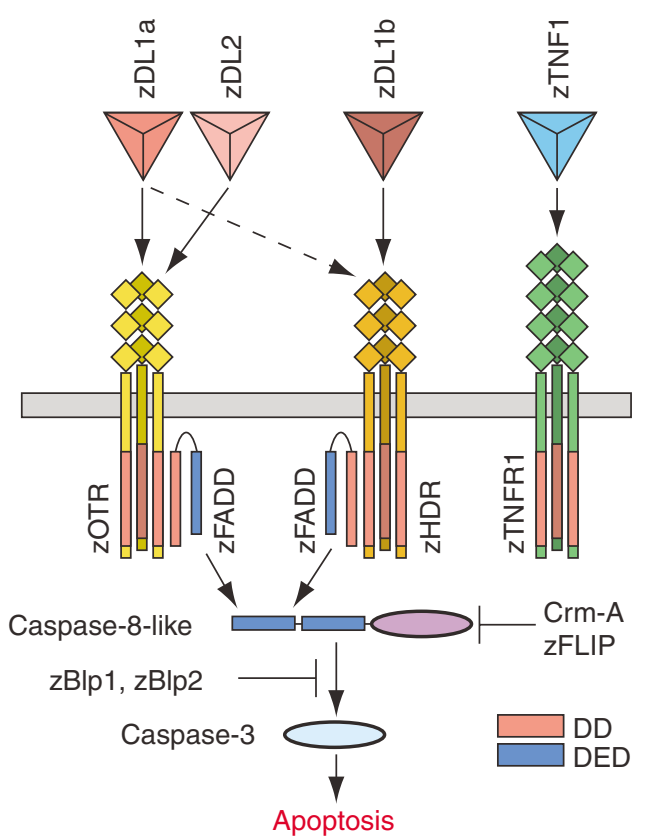

Figure 9 Schematic representation of established ligand/receptor interactions and the intracellular machinery required for effector caspase activation in zebrafish. zDL1a binds both zHDR and zOTR in vitro but signals exclusively through zOTR when overexpressed during embryogenesis. Ligand-mediated apoptosis in the notochord and ICM can be blocked by inhibition of of caspase-8-like proteases (using CrmA or zFLIP) or the cell-intrinsic apoptosis pathway (using zBlp1 or zBlp2) from those of the human Apo2L/TRAIL DD-containing receptors DR4 and DR5 and the decoy receptors DcR1, DcR2, and osteoprotegerin. ${ }^{43,44}$ Thus, receptors for this ligand do not necessarily share a conserved ECD architecture in nonmammalian species.

Mice lacking FADD, c-FLIP, or caspase-8 develop abnormal cardiac morphology between embryonic days 9.5 and 10.5 and die soon thereafter. ${ }^{11,13,14}$ Zebrafish embryos reach equivalent stages of heart development by $\sim 48$ h.p.f. ${ }^{45}$ Various techniques for inhibiting the extrinsic pathway caused no cardiac defects or other morphological abnormalities in zebrafish embryos. The finding that embryonic defects in caspase- 8 deficient mice can be rescued by ex vivo wholeembryo culture suggests that the primary requirement for caspase-8 in mammalian embryogenesis may be in extraembryonic tissues rather than the embryo proper. ${ }^{14}$ Our analysis of the zebrafish, which develops ex utero and lacks extraembryonic tissues, supports the hypothesis that deficiencies in the extrinsic pathway impact very few, if any, tissues in the embryo proper.

To our knowledge, this is the first report that examines the sensitivity of embryonic tissues to ligand-induced apoptosis in any chordate. We found that full-length, transmembrane Apo2L/TRAIL homologs selectively induced apoptosis in the notochord and the ICM of zebrafish embryos. The observation that $z D L 1 b$ overexpression induces apoptosis in embryonic erythroblasts is particularly intriguing, given that inhibition of zHDR stimulates erythropoiesis in adult zebrafish ${ }^{24}$ and 
caspase-8 deficient mice exhibit hyperemia during embryogenesis. ${ }^{10}$ Although we did not see defects in notochord development upon inhibition of the extrinsic pathway, the complementary expression patterns of $z H D R$ and $z O T R$ in the notochord and of $z D L 1 a$ in the surrounding cells is intriguing. Perhaps $z H D R, z O T R$, and $z D L 1 a$ prime the notochord to respond to as yet unidentified environmental cues during embryogenesis.

\section{Materials and Methods}

Data are provided as Supplementary Material.

\section{Acknowledgements}

We thank Dr. Leon Parker for guidance with zebrafish embryology, Dr. Andreas Strasser for insightful discussions, Drs. Richard Harland and Sharon Amacher for materials, and members of the Genentech Molecular Oncology Department for helpful comments and technical advice.

\section{References}

1. Danial NN and Korsmeyer SJ (2004) Cell death: critical control points. Cell 116: 205-219.

2. Puthalakath $\mathrm{H}$ and Strasser $\mathrm{A}$ (2002) Keeping killers on a tight leash: transcriptional and post-translational control of the pro-apoptotic activity of BH3-only proteins. Cell Death Differ. 9: 505-512.

3. Ashkenazi A and Dixit VM (1998) Death receptors: signaling and modulation. Science 281: 1305-1308

4. Ashkenazi A (2002) Targeting death and decoy receptors of the tumournecrosis factor superfamily. Nat. Rev. Cancer 2: 420-430.

5. Igaki $\mathrm{T}$, Kanda $\mathrm{H}$, Yamamoto-Goto $\mathrm{Y}$, Kanuka $\mathrm{H}$, Kuranaga $\mathrm{E}$ and Aigaki $\mathrm{T}$ et al. (2002) Eiger, a TNF superfamily ligand that triggers the Drosophila JNK pathway. EMBO J. 21: 3009-3018.

6. Kanda H, Igaki T, Kanuka H, Yagi T and Miura M (2002) Wengen, a member of the Drosophila tumor necrosis factor receptor superfamily, is required for Eiger signaling. J. Biol. Chem. 277: 28372-28375.

7. Brojatsch J, Naughton J, Adkins HB and Young JA (2000) TVB receptors for cytopathic and noncytopathic subgroups of avian leukosis viruses are functional death receptors. J. Virol. 74: 11490-11494.

8. Varfolomeev EE and Ashkenazi A (2004) Tumor necrosis factor: an apoptosis JuNKie? Cell 116: 491-497.

9. Locksley RM, Killeen N and Lenardo MJ (2001) The TNF and TNF receptor superfamilies: integrating mammalian biology. Cell 104: 487-501.

10. Varfolomeev EE, Schuchmann M, Luria V, Chiannlkulchai N, Beckmann JS and Mett IL et al. (1998) Targeted disruption of the mouse caspse-8 gene ablates cell death induction by the TNF receptors, Fas/Apo1, and DR3 and is lethal prenatally. Immunity 9: 267-276.

11. Yeh WC, de la Pompa JL, McCurrach ME, Shu HB, Elia AJ and Shahinian A et al. (1998) FADD: essential for embryo development and signaling from some, but not all, inducers of apoptosis. Science 279: 1954-1958.

12. Zhang J, Cado D, Chen A, Kabra NH and Winoto A (1998) Fas-mediated apoptosis and activation-induced T-cell proliferation are defective in mice lacking FADD/Mort1. Nature 392: 296-300.

13. Yeh W-C, Itie A, Elia A, Ng M, Shu H-B and Wakeham A et al. (2000) Requirement for Casper (c-FLIP) in regulation of death receptor-induced apoptosis and embryonic development. Immunity 12: 633-642.

14. Sakamaki K, Inoue T, Asano M, Sudo K, Kazama H and Sakagami J et al. (2002) Ex vivo whole-embryo culture of caspase-8-deficient embryos normalize their aberrant phenotypes in the developing neural tube and heart. Cell Death Differ. 9: 1196-1206.

15. Cretney E, Takeda K, Yagita H, Glaccum M, Peschon JJ and Smyth MJ (2002) Increased susceptibility to tumor initiation and metastasis in TNF-related apoptosis-inducing ligand-deficient mice. J. Immunol. 168: 1356-1361.
16. Karray S, Kress C, Cuvellier S, Hue-Beauvais C, Damotte D and Babinet $C$ et al. (2004) Complete loss of Fas ligand gene causes massive lymphoproliferation and early death, indicating a residual activity of gld allele. J. Immunol. 172: 2118-2125.

17. Schmaltz C, Alpdogan O, Kappel BJ, Muriglan SJ, Rotolo JA and Ongchin J et al. (2002) T cells require TRAIL for optimal graft-versus-tumor activity. Nat. Med. 8: 1433-1437.

18. Lamhamedi-Cherradi SE, Zheng SJ, Maguschak KA, Peschon J and Chen YH (2003) Defective thymocyte apoptosis and accelerated autoimmune diseases in TRAIL-/- mice. Nat. Immunol. 4: 255-260.

19. Inohara $N$ and Nunez $G$ (2000) Genes with homology to mammalian apoptosis regulators identified in zebrafish. Cell Death Differ. 7: 509-510.

20. Pressley ME, Phelan III PE, Witten PE, Mellon MT and Kim CH (2005) Pathogenesis and inflammatory response to Edwardsiella tarda infection in the zebrafish. Dev. Comp. Immunol. 29: 501-513.

21. Savan R, Kono T, Igawa D and Sakai M (2005) A novel tumor necrosis factor (TNF) gene present in tandem with theTNF-alpha gene on the same chromosome in teleosts. Immunogenetics 57: 140-150.

22. Bobe J Goetz FW (2001) Molecular cloning and expression of a TNF receptor and two TNF ligands in the fish ovary. Comp. Biochem. Physiol. B Biochem. Mol. Biol. 129: 475-481.

23. Kwan TT, Liang R, Verfaillie CM, Ekker SC, Chan LC and Lin S et al. (2006) Regulation of primitive hematopoiesis in zebrafish embryos by the death receptor gene. Exp. Hematol. 34: 27-34.

24. Long Q, Huang H, Shafizadeh E, Liu N and Lin S (2000) Stimulation of erythropoiesis by inhibiting a new hematopoietic death receptor in transgenic zebrafish. Nat. Cell Biol. 2: 549-552.

25. Talbot WS, Trevarrow B, Halpern ME, Melby AE, Farr G and Postlethwait JH et al. (1995) A homeobox gene essential for zebrafish notochord development. Nature 378: 150-157.

26. Halpern ME, Ho RK, Walker C and Kimmel CB (1993) Induction of muscle pioneers and floor plate is distinguished by the zebrafish no tail mutation. Cell 75: 99-111.

27. Al-Adhami MA and Kunz YW (1977) Ontogenesis of haematopoietic sites in Brachydanio rerio (Hamilton-Buchanan) (Teleostei). Develop. Growth Different. 19: $171-179$

28. Dijkgraaf $S$ (1963) The functioning and significance of the lateral-line organs. Biol. Rev. Camb. Philos. Soc. 38: 51-105.

29. Metcalfe WK (1989) Organization and development of the zebrafish posterior lateral line In: Coombs S, Görner P, Münz H (eds). The Mechanosensory Lateral Line. Springer-Verlag: New York pp 147-159.

30. Haddon C and Lewis J (1996) Early ear development in the embryo of the zebrafish, Danio rerio. J. Comp. Neurol. 365: 113-128.

31. Williams JA and Holder N (2000) Cell turnover in neuromasts of zebrafish larvae. Hear Res. 143: 171-181.

32. Sharp DA, Lawrence DA and Ashkenazi A (2005) Selective knockdown of the long variant of cellular FLICE inhibitory protein augments death receptormediated caspase-8 activation and apoptosis. J. Biol. Chem. 280: 1940119409.

33. Srinivasula SM, Ahmad $M$, Fernandes-Alnemri $T$, Litwack $G$ and Alnemri ES (1996) Molecular ordering of the Fas-apoptotic pathway: the Fas/APO-1 protease Mch5 is a CrmA-inhibitable protease that activates multiple Ced-3/ICE-like cysteine proteases. Proc. Natl. Acad. Sci. USA 93: 14486-14491.

34. Garcia-Calvo M, Peterson EP, Leiting B, Ruel R, Nicholson DW and Thornberry NA (1998) Inhibition of human caspases by peptide-based and macromolecular inhibitors. J. Biol. Chem. 273: 32608-32613.

35. Ray CA, Black RA, Kronheim SR, Greenstreet TA, Sleath PR and Salvesen GS et al. (1992) Viral inhibition of inflammation: cowpox virus encodes an inhibitor of the interleukin- 1 beta converting enzyme. Cell 69: 597-604.

36. Barnhart BC, Alappat EC and Peter ME (2003) The CD95 type I/type II model. Semin. Immunol. 15: 185-193.

37. Chen MC, Gong HY, Cheng CY, Wang JP, Hong JR and Wu JL (2001) Cloning and characterization of zfBLP1, a Bcl-XL homologue from the zebrafish, Danio rerio. Biochim. Biophys. Acta 1519: 127-133.

38. Langenau DM, Jette C, Berghmans S, Palomero T, Kanki JP and Kutok JL et al. (2005) Suppression of apoptosis by bcl-2 overexpression in lymphoid cells of transgenic zebrafish. Blood 105: 3278-3285. 
39. Davidson AJ and Zon LI (2004) The 'definitive' (and 'primitive') guide to zebrafish hematopoiesis. Oncogene 23: 7233-7246.

40. Detrich III HW, Kieran MW, Chan FY, Barone LM, Yee K and Rundstadler JA et al. (1995) Intraembryonic hematopoietic cell migration during vertebrate development. Proc. Natl. Acad. Sci. USA 92: 10713-10717.

41. Hymowitz SG, O'Connel MP, Ultsch MH, Hurst A, Totpal K and Ashkenazi A et al. (2000) A unique zinc-binding site revealed by a high-resolution X-ray structure of homotrimeric Apo2L/TRAIL. Biochemistry 39: 633-640.
42. Amores A, Force A, Yan YL, Joly L, Amemiya C and Fritz A et al. (1998) Zebrafish hox clusters and vertebrate genome evolution. Science 282: 1711-1714.

43. Ashkenazi A and Dixit VM (1999) Apoptosis control by death and decoy receptors. Curr. Opin. Cell. Biol. 11: 255-260.

44. Bodmer JL, Schneider P and Tschopp J (2002) The molecular architecture of the TNF superfamily. Trends Biochem. Sci. 27: 19-26.

45. Fishman MC and Chien KR (1997) Fashioning the vertebrate heart: earliest embryonic decisions. Development 124: 2099-2117.

Supplementary Information accompanies the paper on Cell Death and Differentiation website (http://www.nature.com/cdd) 